The loss of cyclooxygenase in platelets, which lack the capacity for new protein synthesis, constitutes a loss of function for the remainder of their lifetime. The average life span of platelets is 7 days; therefore the conventional practice is to stop aspirin therapy 1 week before the planned procedure. However, the results of this study show that inhibition of platelet aggregation with aspirin vanishes earlier than expected. The life span of the platelet and platelet aggregation as a whole need to be understood separately. Although it may take 10 days for the total platelet population to be renewed and thus restore normal cyclooxygenase activity, it has been shown in vitro that if as few as $20 \%$ of total platelets have normal cyclooxygenase activity, aggregation would remain normal. ${ }^{5}$ Our study has confirmed this finding in the clinical situation.

This study has several limitations. One of these is the small study population. We therefore should extend this study and investigate the outcomes. Another limitation concerns aspirin dosages, which were low $(81 \mathrm{mg} / \mathrm{d})$ in this study. If the aspirin dosage had been higher, the speed of recovery of aggregation might have been different.

In conclusion, this study indicates that inhibition of platelet aggregation with an aspirin fell quickly at 1 day after discontinu- ation and vanished after 3 days. Therefore, in the presence of preoperative aspirin therapy, stopping at 3 days before surgery might be optimal for preventing bleeding complications. In addition, this study result should be considered when comparing aspirin users versus nonusers in future investigations into the effects of aspirin in cardiac surgery.

\section{References}

1. Sethi GK, Copeland JG, Goldman S, Moritz T, Zandina K, Henderson WG, et al. Implications of preoperative administration of aspirin in patients undergoing coronary artery bypass grafting. J Am Coll Cardiol. 1990; 15:15-20.

2. Dacey LJ, Munoz JJ, Johnson ER, Leavitt BJ, Maloney CT, Morton JR, et al. Effect of preoperative aspirin use on mortality in coronary artery bypass grafting patients. Ann Thorac Surg. 2000;70:1986-90.

3. Mase K, Yanagihara F, Asada T, Yamanaka Y, Yoshioka M, Yasunaga K. Evaluation of platelet aggregation test by grading curve. Jpn J Clin Pathol. 1992;40:857-62.

4. Borns CV, Cross MJ. The aggregation of blood platelets. J Physiol. 1963;168:178-95.

5. Bradlow BA, Chetty N. Dosage frequency for suppression of platelet function by low dose aspirin therapy. Thromb Res. 1982;27:99-110.

\title{
Surgical pitfalls during explantation of a thoracic aortic endoprosthesis
}

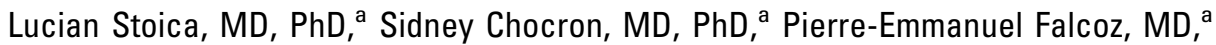 \\ Jean-François Bonneville, MD, ${ }^{b}$ and Joseph-Philippe Etievent, MD, ${ }^{a}$ Besançon, France
}

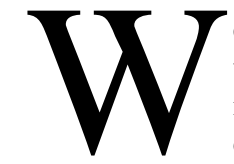

e report our experience with explantation of a thoracic aortic endoprosthesis 2 years after the initial procedure. This graft was explanted because of failure of endovascular treatment of an aneurysm that developed after surgical treatment of a type A aortic dissection. Crossclamping of the aortic arch was impossible, and surgery had to be performed with the patient under circulatory arrest. The procedures performed are time-consuming under circulatory arrest, making distal aortic arch surgery more complex after endovascular repair than when performed at primary surgery.

\footnotetext{
From the Departments of Thoracic and Cardio-Vascular Surgery ${ }^{\mathrm{a}}$ and

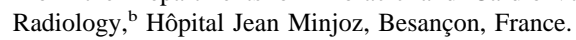

Received for publication Dec 13, 2003; accepted for publication Dec 30, 2003.

Address for reprints: Lucian Stoica, MD, PhD, Department of Thoracic and Cardiovascular Surgery, Hôpital Jean Minjoz, 3 Blvd Fleming, Besançon, France 25000 (E-mail: 1.stoica@ voila.fr).

J Thorac Cardiovasc Surg 2004;127:1815-7

$0022-5223 / \$ 30.00$

Copyright $\odot 2004$ by The American Association for Thoracic Surgery

doi:10.1016/j.jtcvs.2003.12.026
}

\section{Clinical Summary}

A 48-year-old man was operated on for acute type-A aortic dissection. We performed a supracoronary graft replacement of the ascending aorta under hypothermic circulatory arrest with cerebral retroperfusion through the superior vena cava. ${ }^{1}$ We found only one intimal tear on the ascending aorta. The aortic valve was spared. One year later, magnetic resonance imaging control revealed a perfused false lumen with dilatation of the aorta to 53 $\mathrm{mm}$. Aortography showed a communication between the two lumens just below the origin of the left subclavian artery. A Talent endoluminal stent graft (World Medical Corp, Sunrise, Fla) was deployed 17 months after the first operation. It covered the origin of the left subclavian artery, and the uncovered part was placed facing the origin of the left common carotid artery. Because the false lumen was still being perfused by the left subclavian artery, the origin of the artery was completely obstructed with coils (Figure 1). The treatment was completed with a bypass between the left carotid artery and the left subclavian artery to avoid left arm ischemia.

Two years after endovascular treatment, the false lumen was still perfused and the diameter of the postisthmic thoracic aorta had increased to $57 \mathrm{~mm}$ (Figure 1). We performed the explantation of the endoprosthesis through a left thoracotomy with the patient 

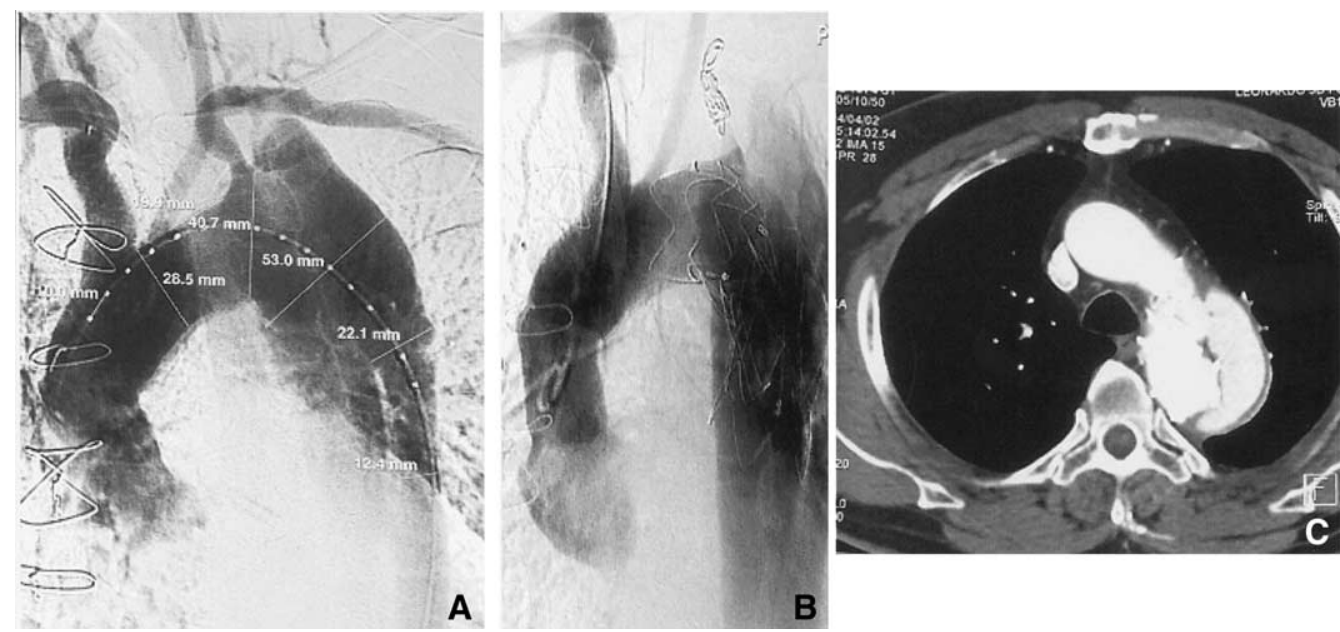

Figure 1. False lumen is still perfused after endovascular treatment. Arteriography of initial aneurysm $(A)$ and after stent-graft deployment (B), and computed tomographic scan $\mathbf{2 0}$ months after endovascular treatment (C). Thoracic aorta developed life-threatening aneurysm.
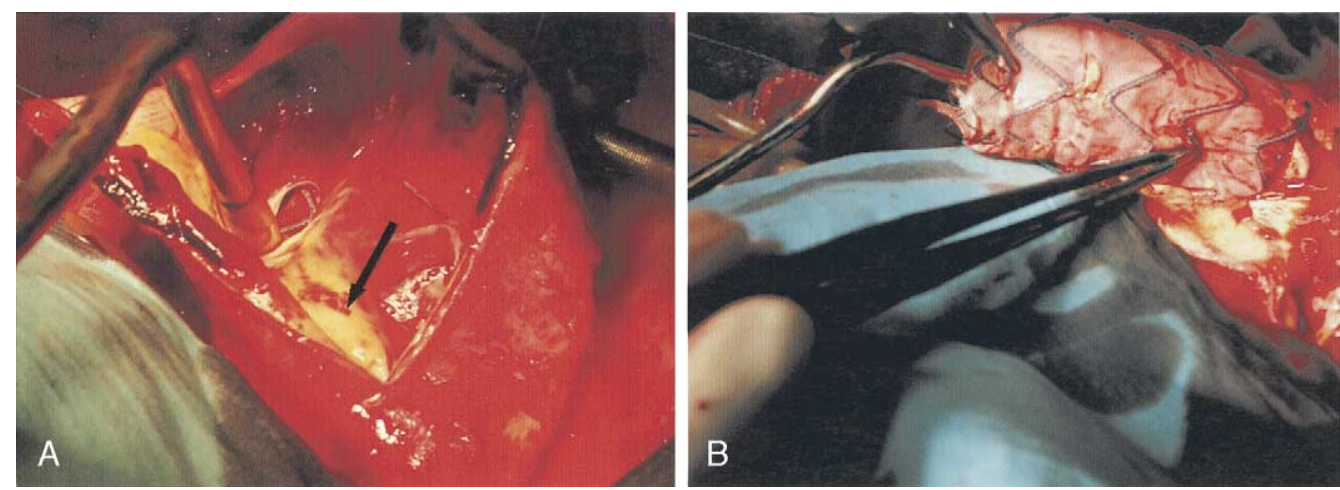

Figure 2. A, Cannula passes through communication between false lumen (arrow) and true lumen containing stent graft. B, Endoprosthesis inside true lumen.

under hypothermic circulatory arrest, and we replaced the proximal part of the descending aorta with a Dacron polyester fabric graft. Left femoral vein cannulation was impossible, so the pulmonary artery trunk was cannulated to drain the venous blood. The arterial blood was injected into the left femoral artery. Cerebrospinal fluid drainage was used to enhance medullar protection. ${ }^{2}$ While opening the false lumen longitudinally, we found that the aortic stent did not adhere to the aortic wall in the Dacron graftcovered part; rather, the two extremities not covered were deeply embedded in the aortic media and hampered stent extraction. We also found a large communication between the two channels at the level of the proximal uncovered part of the stent (Figure 2). Finally, the aortic arch was not dissected, and the aneurysm started at the level of the left subclavian artery, the origin of which was totally thrombosed.

A 30-mm Dacron polyester fabric graft was inserted from the heel of the left common carotid artery down to the sixth dorsal vertebra level. Distally, the graft anastomosis reimplanted both channels. The patient was doing well 10 months after this operation.

\section{Discussion}

In our experience, surgery of the distal aortic arch and proximal descending aorta is more difficult after endovascular treatment than when performed in primary surgery. Crossclamping of the aortic arch in this case was impossible because the uncovered part of the endoprosthesis was placed inside the aortic arch, facing the left carotid artery ostia. This meant that surgery had to be done with the patient under circulatory arrest.

The explantation of the endoprosthesis was difficult. The graft became embedded in the media of the aortic wall, so we had to be cautious when extracting the two extremities of the endoprosthesis. There were only a few centimeters of aortic arch between the distal suture of the ascending aorta Dacron polyester fabric graft and the proximal extremity of the endoprosthesis. Because of the high risk 
of aortic arch rupture, we could not remove the covered portion of the endoprosthesis by pulling it. We had to cut and remove each branch separately, which extended the duration of circulatory arrest significantly. The proximal suture of the arch reconstruction was difficult to perform because of fibrosis of the tissues.

In our case femoral vein cannulation was impossible; the cannula stopped in the iliac vein with the patient in the left thoracotomy position as well as the dorsal decubitus position. A good venous return is needed for circulatory arrest, so we cannulated the pulmonary artery trunk This kind of cannulation entails considerable risk because of the fragility of the tissue, and the pulmonary artery may be torn when cannulated. Moreover, occluding the cannula to avoid leakage of blood is difficult.

We found a large communication between the two channels facing the proximal uncovered part of the stent. We do not know whether this communication existed before or was caused by the insertion of the stent, but it was the cause of failed endovascular treatment in our case. We did not use a balloon to expand the stent when deploying the endoprosthesis to avoid any risk of creating a communication between the two channels.

These findings suggest the difficulties associated with the endovascular repair of the dissections. In chronic dissection, multiple reentry points between the two lumina may be found on the descending thoracic aorta, making endovascular treatment hazardous. If secondary surgery is required, the repair is more difficult than it is in primary surgery because aortic crossclamping is impossible. Therefore the explantation of the endoprosthesis and the reconstruction of the aortic arch must be done under circulatory arrest. The explantation of the stent graft is also technically difficult, because it is deeply embedded in the aortic wall.

We thank Nancy Richardson-Peuteuil for her editorial assistance.

\section{References}

1. Ueda Y, Miki S, Okita Y, Tahata T, Ogino H, Sakai T, et al. Protective effect of continuous retrograde cerebral perfusion on the brain during deep hypothermic systemic circulatory arrest. J Card Surg. 1994;9:58495.

2. Coselli JS, Conklin LD, LeMaire SA. Thoracoabdominal aortic aneurysm repair: review and update of current strategies. Ann Thorac Surg. 2002;74:S1881-4.

\title{
Should heart transplantation be considered as a treatment option for patients aged 70 years and older?
}

\author{
Jeffrey A. Morgan, MD, Ranjit John, MD, Donna M. Mancini, MD, and Niloo M. Edwards, MD, New York, NY
}

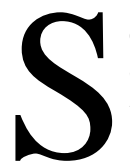

everal studies have demonstrated favorable results after orthotopic cardiac transplantation in recipients of advanced age. ${ }^{1-4}$ These studies have demonstrated similar survival in elderly patients as compared to that of younger patients undergoing heart transplantation. This has resulted in a relaxation of the upper age limit for cardiac transplantation in many centers. However, some transplant centers continue to view advanced recipient age as a contraindication to transplantation because of concerns regarding decreased survival in this cohort. ${ }^{5-7}$ This study was conducted to analyze and compare our experiences with older (70 years and older) versus younger

From the Department of Surgery, Division of Cardiothoracic Surgery, College of Physicians and Surgeons, Columbia University, New York, NY.

Received for publication Nov 27, 2003; accepted for publication Dec 16, 2003.

Address for reprints: Jeffrey A. Morgan, MD, Columbia University, College of Physicians and Surgeons, 177 Fort Washington Ave, Milstein Hospital 7GN-435, New York, NY 10032 (E-mail: jm2240@columbia. edu).

J Thorac Cardiovasc Surg 2004;127:1817-9

$0022-5223 / \$ 30.00$

Copyright $\odot 2004$ by The American Association for Thoracic Surgery

doi:10.1016/j.jtcvs.2003.12.035 (younger than 70 years) recipients with respect to perioperative and long-term survivals.

\section{Patients and Methods}

Between January 1992 and February 2003, a total of 939 orthotopic cardiac transplants were performed at the New York Presbyterian Hospital-Columbia Medical Center, New York. Of these, $10(1.1 \%)$ were performed in patients 70 years old or older (range 70.2-74.8 years). These patients were compared with patients younger than 70 years (range 18.3-69.8 years) at the time of transplantation. Pediatric patients (younger than 18 years; $n=$ 115) were excluded. Outcome measures included perioperative and long-term posttransplantation survivals.

Statistical analysis. Data were represented as frequency distributions and percentages. Values of continuous variables were expressed as mean $\pm \mathrm{SD}$. Continuous variables were compared with paired $t$ tests, whereas categorical variables were compared by means of $\chi^{2}$ tests. Kaplan-Meier analysis was used to calculate survival along with a log-rank $P$ value when comparing groups. Actuarial survivals at $1,3,5$, and 10 years after transplantation were calculated by constructing life tables. Significant predictors of mortality were identified with multivariate Cox proportional hazard models. All data were analyzed with the SPSS 11.5 software package (SPSS Inc, Chicago, Ill). 